\title{
Stress Effects on Magnetic Properties of Amorphous Microwires Subjected to Current Annealing
}

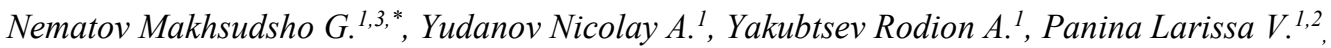 \\ Beklemisheva Anna V. ${ }^{1}$, Morchenko Alexander T. ${ }^{1}$, Thakur Atul ${ }^{4}$ \\ ${ }^{1}$ National University of Science and Technology, MISIS, Moscow 119991, Russia \\ ${ }^{2}$ Institute for Design Problems in Microelectronics RAS, Moscow 124681, Russia \\ ${ }^{3}$ Tajik Technical University named after ac. M.S. Osimi, Dushanbe, 734042, Tajikistan \\ ${ }^{4}$ Centre of Nanotechnology, Amity, University Haryana, Manesar-122413, India
}

\begin{abstract}
Effects of current annealing on magnetic hysteresis properties and magnetoimpedance (MI) of glass-coated amorphous microwires with nominal composition of $\mathrm{Co}_{71} \mathrm{Fe}_{5} \mathrm{~B}_{11} \mathrm{Si}_{10} \mathrm{Cr}_{3}$ and small positive magnetostriction were investigated with the purpose to control the magnetic anisotropy. We have demonstrated that current annealing can produce a controllable change in the easy anisotropy direction from almost axial to circular, depending on the annealing time. The induced magnetization configuration is very sensitive to the applied tensile stress. The combination of positive magnetostriction and helical anisotropy makes it possible to realise large stress-magnetoimpedance (S-MI) effect without use of any dc bias fields owing to the directional change in magnetization. This is especially important for microwave frequency S-MI effect and can be very interesting for developing stress-sensors operating at the high frequency region.
\end{abstract}

\section{Introduction}

In recent years, a considerable interest has been devoted to studying the magnetization processes and magnetoimpedance (MI) in Co-Fe based amorphous glass-coated microwires (diameter is between 1 to 100 $\mu \mathrm{m})$, which is related to their technical applications in miniature sensor devices [1-4]. Owing to the absence of a crystalline structure the magnetoelastic interactions play the main role in determining the magnetic anisotropy in amorphous microwires. The easy anisotropy axes are therefore affected by the value of the saturation magnetostriction and the distribution and values of internal stresses both of which may strongly depend on temperature. This could be useful to modify the magnetic anisotropy by various heat treatments partially releasing the internal stress occurring during fast solidification [57]. Among thermal treatments annealing in the presence of magnetic field has a special importance since it is possible to form easy anisotropy direction along the field due to short-range pair ordering [8-9].

$\mathrm{Co}-\mathrm{Fe}$ alloys on Co-rich side possess a near-zero magnetostriction and excellent soft magnetic properties. In these systems, it is possible to control the magnetic anisotropy by current annealing which combines the Joule heating and circular magnetic field [10-12]. This technique also helps to avoid the deviations in the easy anisotropy due to inhomogeneous distribution of internal stresses. In microwires with a small negative magnetostriction where the magnetoelastic anisotropy is predominantly circumferential the current annealing helps to improve this anisotropy and to enhance the relative change in impedance up to $600 \%$ [10].

In the present work, we report on the influence of dc current annealing on the magnetic anisotropy in Co-rich amorphous microwires having a small positive magnetostriction. We have demonstrated that the current annealing produces a controllable change in the easy anisotropy direction from almost axial to helical accompanied by corresponding changes in the magnetic hysteresis and MI. The induced helical anisotropy not only increases the relative change in impedance (MI ratio) up to $200 \%$ (from $10 \%$ in as-prepared state) in the lowfield region, but makes it possible to realize highly stresssensitive MI. This has a potential for applications in miniature stress sensors, in particular, for embedded sensing techniques [13-15].

\section{Analysis of induced anisotropies}

In amorphous alloys, the effective magnetocrystalline anisotropy is low due to the averaging effect of exchange interaction. The additional contribution to the anisotropy before annealing comes from magnetoelastic interactions. In glass-coated amorphous wires the internal stress $\sigma_{i}$ originated from quenching and drawing processes is predominantly tensile and directed along the wire [15-18].

The combination of a negative magnetostriction and tensile stress produces a circumferential easy anisotropy. Then, the external tensile stress strengthens this anisotropy, but the character of magnetization loops does not change. This could present a problem when realizing

\footnotetext{
* Corresponding author: nematovmaqsud@misis.ru
} 
stress-sensitive MI, since the highest sensitivity of the impedance change is caused by the directional change in magnetization [19-20].

For positive magnetostriction the tensile stress sets the axial easy anisotropy. Heat treatments produce easy anisotropies along the direction of local magnetization. The presence of a magnetic field during annealing aligns the magnetization and induces a uniform anisotropy with the easy axis along the field, that is, along circumference. In this case, the applied tensile stress changes the direction of the easy axis which is needed for large changes in impedance, especially at microwave frequencies [13].

To analyze the change in the magnetic anisotropy due to current annealing and application of external stresses $\sigma_{e x}$ it is assumed that the wire has also a frozen-in torsion stress $\sigma_{t}$, that corresponds to the effects of tension and compression $\pm \sigma_{t}$ directed at $90^{\circ}$ to each other and at $45^{\circ}$ with respect to the wire axis. The presence of torsion will allow us to introduce a generalized helical magnetic anisotropy and to describe a gradual transition from axial to circular anisotropy due to annealing and from circular to axial anisotropy after annealing upon the application of an external tensile stress. The current annealing induces a circumferential easy anisotropy the value of which $K_{a}$ depends on the annealing conditions. The magnetic energy $U_{m}$ can be then written in the form:

$$
\begin{gathered}
U_{m}=-\left(K-K_{a}\right) \cos ^{2} \theta-\frac{3}{2} \lambda\left(\sigma_{i}+\sigma_{e x}\right) \cos ^{2} \theta- \\
\frac{3}{2} \lambda \sigma_{t}\left[\cos ^{2}\left(\theta-\frac{\pi}{4}\right)-\cos ^{2}\left(\theta+\frac{\pi}{4}\right)\right]
\end{gathered}
$$

Here $K$ is the uniaxial anisotropy constant, which is not related to the magnetostriction interactions, $\theta$ is the angle between the magnetization and the wire axis, and $\lambda>0$ is the magnetostriction coefficient. Equation (1) can be converted into the form typical of a uniaxial anisotropy with the easy anisotropy axis defined by angle $\alpha$ with respect to the wire axis:

$$
U_{m}=-|\widetilde{K}| \cos ^{2}(\alpha-\theta)
$$

The equivalent constant of uniaxial anisotropy $\widetilde{K}$ is defined as :

$$
\begin{aligned}
& \widetilde{K}=\left[K-K_{a}+\left(\frac{3}{2}\right) \lambda\left(\sigma_{i}+\sigma_{e x}\right)\right] / \cos 2 \tilde{\alpha} \\
& \tilde{\alpha}=\frac{1}{2} \tan ^{-1} \frac{3\left|\lambda \sigma_{t}\right|}{\left|K-K_{a}+(3 / 2) \lambda\left(\sigma_{i}+\sigma_{e x}\right)\right|}
\end{aligned}
$$

The easy angle $\alpha$ in equation (2) depends on the sign of $\widetilde{K}: \alpha=\tilde{\alpha}$ for $\widetilde{K} \geq 0$ and $\alpha=90^{\circ}-\tilde{\alpha}$ for $\widetilde{K}<0$.

In the case of $K>0$ and a positive magnetostriction $\lambda>0$, before annealing the angle $\alpha<45^{\circ}$ and the easy anisotropy axis is close to the wire axis, if $\sigma_{i} \gg \sigma_{t}$. Upon annealing, there is an increasing contribution of $K_{a}$ so the sign of $\widetilde{K}$ becomes negative which allignes the anisotropy closer to circumference $\left(\alpha>45^{\circ}\right)$. This will change the character of the magnetization curves: the rectangular loop is transformed into an inclined flat loop, and the hysteresis region decreases significantly. Upon the application of external tensile stresses, the effective anisotropy constant $\widetilde{K}$ becomes positive and the anisotropy direction tends towards the wire axis $(\alpha<$ $\left.45^{\circ}\right)$.

\section{Experimental details}

Amorphous glass-coated microwires with the nominal composition of $\mathrm{Co}_{71} \mathrm{Fe}_{5} \mathrm{~B}_{11} \mathrm{Si}_{10} \mathrm{Cr}_{3}$ produced by the Taylor-Ulitovski technique [21] were kindly supplied by MFTI Ltd. (Republic of Moldova). The total diameter was $29.5 \mu \mathrm{m}$ and the metal core diameter was about $23.9 \mu \mathrm{m}$. The insulating glass was of Pyrex type. The wires have a small positive magnetostriction in the order of $10^{-7}$.

The Curie temperature $\left(\mathrm{T}_{\mathrm{c}}=637 \mathrm{~K}\right)$ and the crystallization temperature $\left(\mathrm{T}_{\mathrm{cr}}=736 \mathrm{~K}\right)$ were estimated from DSC curves (by using the DSC 204 F1 Netzsch instrumentation) with the help of standard IT application. The DSC curves were also measured after annealing confirming that the amorphous structure did not undergo noticeable changes.

The wire samples of $15 \mathrm{~cm}$ long were annealed by passing a dc current $(I)$ of $50 \mathrm{~mA}$ during 1 - 3 hours in air. All current treatments were performed in the same ambient conditions. The current magnitude was chosen in order to realize a moderate heating effect in the range of temperatures 470 - $530 \mathrm{~K}$, which are considerably lower than $\mathrm{T}_{\mathrm{c}}$ and $\mathrm{T}_{\mathrm{cr}}$. The electrical contacts were made by standard soldering technique removing the glass coat at the ends. The current $(I)$ produces a circular magnetic field $H_{a}$ the value of which at the sutface $H_{a}=I / 2 \pi a$ was about $400 \mathrm{~A} / \mathrm{m}$ which is sufficient to align the local magnetization.

The hysteresis loops under the effect of applied load up to $340 \mathrm{MPa}$ were obtained by using induction method with two differential detection/magnetization coils having an inner diameter of $3 \mathrm{~mm}$. The magnetization coil was driven by a current of a frequency of $500 \mathrm{~Hz}$, which produced a magnetizing field with the amplitude of up to $1000 \mathrm{~A} / \mathrm{m}$. Such magnitude is sufficient for remagnetizing the wires. The load was hang at one end of the microwires whilst the other was kept fixed. The magneto-impedance was measured using a HewlettPackard 8753E Vector Network Analyzer in the frequency range between 1 and $100 \mathrm{MHz}$ at room temperature. Two-port measuring scheme $\left(S_{21}\right)$ was used. The sample was placed inside of a solenoid coil that produced a slowly varying magnetic field up to $600 \mathrm{~A} / \mathrm{m}$. In order to introduce a tensile stress during $S_{21}$ measurement, stripes of paper of equal thickness were inserted under the middle part of the wire. It is difficult to evaluate an exact value of the applied stress but it is possible to continuously increase its magnitude by increasing the number of stripes.

\section{Results and discussion}

The longitudinal hysteresis loops for as-cast and current annealed wires are shown in Figure 1. Obviously, the current annealing produced a change in the direction of the easy anisotropy as discussed above. For sufficiently long annealing times (about 2 hours or longer) the current induced anisotropy dominates over the residual 
contributions from the magnetocrystalline and magnetoelastic anisotropies, so the analysis based on equations (1) - (4) can be applied. Accordingly, a near rectangular loop transforms into a flat-type loop and for the annealing time of 2 hours the remanence-to-saturation ratio reduces to about 0.15 . This corresponds to almost a circular easy anisotropy. For longer annealing times the type of magnetic hysteresis does not change but the effective anisotropy continuous to grow. This further increase in the anisotropy field is unfavorable for various sensing applications and an optimal annealing time should be used.

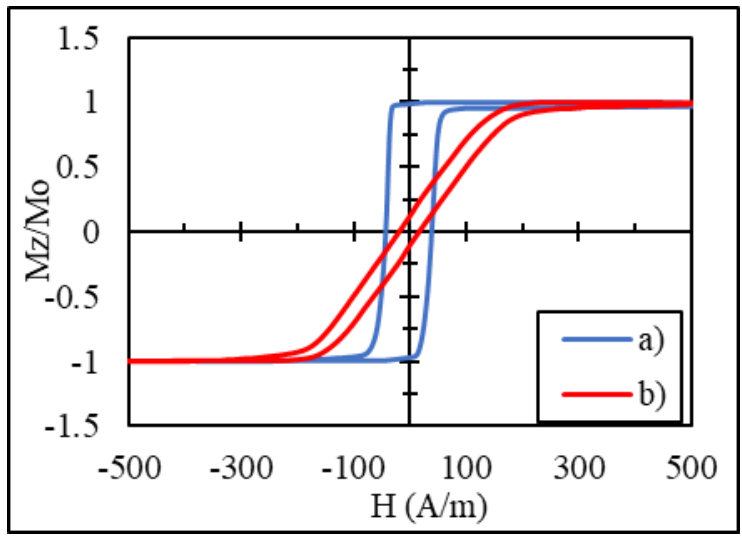

Fig. 1. Hysteresis loops of amorphous $\mathrm{Co}_{71} \mathrm{Fe}_{5} \mathrm{~B}_{11} \mathrm{Si}_{10} \mathrm{Cr}_{3}$ microwires: a) as-prepared and b) after annealing by current of $50 \mathrm{~mA}$ for 2 hours.

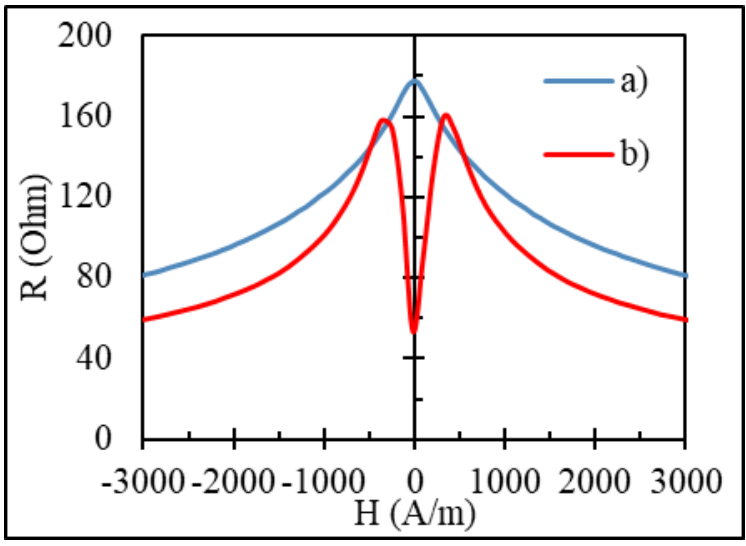

Fig. 2. Real part of the impedance vs. magnetic field at $50 \mathrm{MHz}$ for amorphous $\mathrm{C}_{71} \mathrm{Fe}_{5} \mathrm{~B}_{11} \mathrm{Si}_{10} \mathrm{Cr}_{3}$ microwires: a) as-prepared and $b$ ) after annealing by current of $50 \mathrm{~mA}$ for 2 hours.

The coercivity value continuously decreases with increasing annealing time. For as-prepared wires, $H_{c}$ is about $25 \mathrm{~A} / \mathrm{m}$ whereas it decreases down to $13 \mathrm{~A} / \mathrm{m}$ for annealing time of 2 hours. The coercivity decrease after annealing is associated firstly with a different magnetization configuration: the driving field becomes almost orthogonal with respect to the easy anisotropy axis.

We further investigated the magnetoimpedance characteristics in the frequency range of $1-100 \mathrm{MHz}$. It is interesting to compare the MI behavior in microwires before and after current annealing, which confirms a drastic change in the anisotropy by current annealing. The
MI plots are presented in Figure 2. For the as-prepared wires, the impedance has a maximum in the zero field, which is typical of the systems with the easy anisotropy axis parallel to the high-frequency current and dc external magnetic field. After current annealing, the impedance behavior has changed showing two symmetrical peaks, which is consistent with the transverse anisotropy with respect to the high frequency current and dc magnetic field [19-20].

It is also seen that the MI ratio after current annealing increased: in the low field region $(<400 \mathrm{~A} / \mathrm{m})$, it is about $200 \%$. For as prepared wires, the sensitivity is much lower, about $10 \%$ in the same field region. This indicates that the induced anisotropy constant $K_{a}$ can be tailored to remain small enough and the anisotropy axis can be uniformly oriented in order to achieve high permeability and high sensitivity of MI $[19,22]$. The value of the effective circular anisotropy after current annealing estimated from the impedance plot is about $400 \mathrm{~A} / \mathrm{m}$ which is about twice as large comparing to that found from the hysteresis loops. This suggests that the induced anisotropy may depend on the wire radius: MI reflects the anisotropy in the surface layer whereas the hysteresis is associated with the averaged anisotropy.

The induced anisotropy may be referred to as a reverse anisotropy with respect to the sign of magnetostriction. Customarily, amorphous wires have an axial or circular anisotropy for positive and negative magnetostriction, respectively. In those cases, in order to observe the tensile stress effect on either the magnetization or MI it is necessary to apply a bias current or a bias field, which is not desirable for many applications. Inducing a circular anisotropy in positive magnetostriction wires makes it possible to realize strong influence of the applied tensile stress $\sigma_{e x}$ on both the magnetization behavior and MI. Figure 3 shows the transformation of the hysteresis loop under the effect of $\sigma_{e x}$ : for sufficiently large $\sigma_{e x}$ a perfect rectangular loop is observed. Therefore, the effective anisotropy has the axial easy axis as a result of the magnetoelastic contribution which is consistent with equations (2) - (4).

The MI behavior in the presence of $\sigma_{e x}$ shown in Figure 4 follows the change in the magnetization. As $\sigma_{e x}$ is increasing the two MI peaks gradually merge and finally the MI plot with a single peak at zero field is observed. This happens since the external stress is capable of changing the direction of the static magnetization rotating it towards the axis. The change in impedance by $\sigma_{e x}$ at zero external field is more than $250 \%$ for the highest stress which is estimated to be in the range of 350 $\mathrm{MPa}$. The observed effect can be referred to as a giant stress-magnetoimpedance effect (S-MI).

\section{Conclusion}

The obtained experimental results on Co-based amorphous glass-coated microwires subjected to current annealing and tensile stress indicate the possibility to control the magnetic anisotropy, that is, to establish welldefined easy anisotropy axes in a desirable way whilst keeping the anisotropy value low, so the wires preserve 
soft magnetic properties. This makes it possible to tailor the magnetic hysteresis and magnetoimpedance by small external stimuli. Thus, inducing a circumferential easy anisotropy in positive magnetostriction wires creates the condition for very large impedance change by applying a tensile stress which could be named as giant stressmagnetoimpedance effect (S-MI). The latter has a potential for developing stress-sensitive elements especially for wireless operation at microwave frequencies.

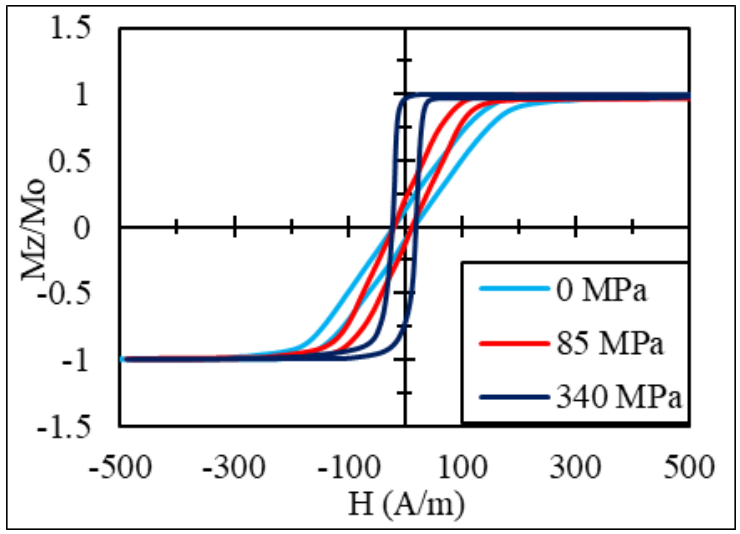

Fig. 3. Effect of tensile stress on the axial hysteresis loops of amorphous $\mathrm{C}_{71} \mathrm{Fe}_{5} \mathrm{~B}_{11} \mathrm{Si}_{10} \mathrm{Cr}_{3}$ microwires after current annealing (50 mA, 2 hours). The load was attached at the free end of the wire.

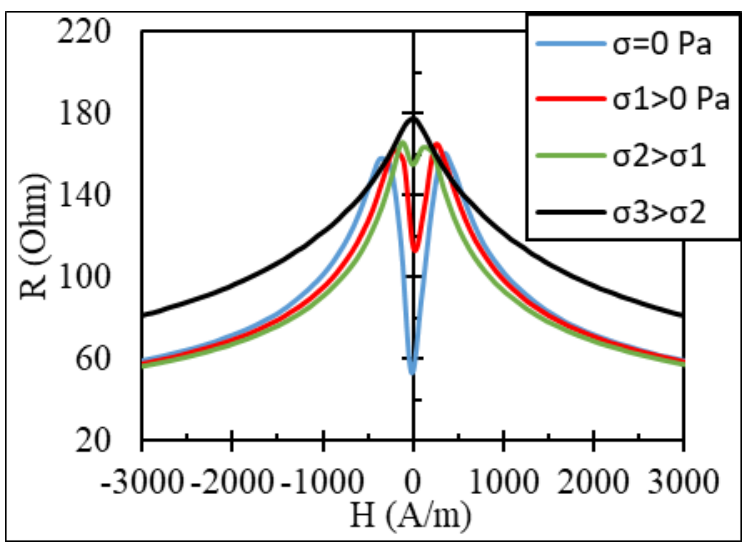

Fig. 4. Effect of tensile stress on the real part of the impedance vs. magnetic field at $50 \mathrm{MHz}$ in amorphous $\mathrm{Co}_{71} \mathrm{Fe}_{5} \mathrm{~B}_{11} \mathrm{Si}_{10} \mathrm{Cr}_{3}$ microwires after current annealing ( $50 \mathrm{~mA}, 2$ hours). The stress is applied by inserting paper-stripes underneath the microwire the both ends of which are soldered at the microstrip cell.

\section{Acknowledgement}

L. V. Panina acknowledges the support for this work under the Russian Federation State Contract for organising a scientific work (grant No 3.8022.2017). The work was also partially supported by the grant from the Ministry of Education and Science of the Russian Federation in the framework of increase Competitiveness Program of NUST "MISIS", implemented by a governmental decree dated 16th of March 2013, No 211.

\section{References}

1. M. Vázquez, H. Chiriac, A. Zhukov, L. Panina and T. Uchiyama, Phys. Status Solidi A, 208, 493 (2011).

2. A. Zhukov and V. Zhukova, Barcelona: International Frequency Sensor Association Publishing, 164, 156 (2013).

3. K. Mohri, T. Uchiyama, L.V. Panina, M. Yamamoto, K. Bushida, J. of Sensors, 2015, 718069 (2015).

4. V.M. García-Chocano, H.G. Miquel, J. Magn. Magn. Mat., 378, 485 (2015).

5. Y. Zhao, H. Hao, Y. Zhang, Intermetallics, 42, 62 (2013).

6. A. Talaat, V. Zhukova, M. Ipatov, J.M. Blanco, L. Gonzalez-Legarreta, B. Hernando, J.J. del Val, J. Gonzalez, A. Zhukov, J. Appl. Phys., 115, 17 A313 (2014).

7. N.A. Yudanov, S.A. Evstigneeva, L.V. Panina, A.T. Morchenko and X.H. Peng, Phys. Status Solidi A, 213, 372 (2016).

8. C. Gomez-Polo and M. Vázquez, J. Magn. Magn. Mat., 118, 86 (1993).

9. D. Mishra, P. Saravanan, A. Perumal and A. Shrinivasan, J. Appl. Phys., 109, 07 A306 (2011).

10. L. Kraus, Z. Frait, K.R. Pirota, H. Chiriac, J. Magn. Magn. Mat., 255, 399 (2003).

11. A. Amirabadizadeh, R. Mardani, M. Ghanaatshoar, J. of Alloys and Comp., 661, 501 (2015).

12. B. Tian, L.H. Wang, L.Y. Zhou, Advanced Materials Research, 79-82, 1407-1410 (2009).

13. D.P. Makhnovskiy, L.V. Panina, C. Garcia, A. Zhukov, J. Gonzalez, Phys. Rev B, 74, 064205 (2006).

14. Y. Luo, F.X. Qin, F. Scarpa, J. Carbonell, M. Ipatov, V. Zhukova, A. Zhukov, J. Gonzalez, L.V. Panina, H.X. Peng, J. Mag. and Mag. Mat., 416, 299 (2016).

15. D. Makhnovskiy, V. Zamorovskii, J. Summerscales, Composites A., 61, 216 (2014).

16. H. Chiriac, T-A. Ovari, A. Zhukov, J. Magn. Magn. Mat., 254-255, 469 (2003).

17. E.E. Shalygina, V.V. Molokanov, M.A. Komarova, J. Experimental and Theoretical Physics, 95, 511 (2002).

18. A.S. Antonov, V.T. Borisov, O.V. Borisov, A.F. Prokoshin and N.A. Usov, J. Phys. D, 33, 1161 (2000).

19. D.P. Makhnovskiy, L.V. Panina, and D.J. Mapps, Phys. Rev. B, 63, 144424 (2001).

20. L.V. Panina, S. I. Sandacci, D.P. Makhnovskiy, J. Appl. Phys., 97, 013701 (2005).

21. A. Zhukov, J. Gonzalez, J.M. Blanco, M. Vázquez and V. Larin, J. Mater. Res. 15, 10 (2000).

22. A.T. Le, M.H. Phan, C.O. Kim, M. Vázquez, H. Lee, N.Q. Hoa, and S.C. Yu, J. of Phys. D. Applied Physics, 40, 4582 (2007). 